\title{
Hereditary spastic paraplegia: from diagnosis to emerging therapeutic approaches
}

\author{
Samuel Shribman, Evan Reid, Andrew H Crosby, Henry Houlden, Thomas T Warner
}

Hereditary spastic paraplegia (HSP) describes a heterogeneous group of genetic neurodegenerative diseases characterised by progressive spasticity of the lower limbs. The pathogenic mechanism, associated clinical features, and imaging abnormalities vary substantially according to the affected gene and differentiating HSP from other genetic diseases associated with spasticity can be challenging. Next generation sequencing-based gene panels are now widely available but have limitations and a molecular diagnosis is not made in most suspected cases. Symptomatic management continues to evolve but with a greater understanding of the pathophysiological basis of individual HSP subtypes there are emerging opportunities to provide targeted molecular therapies and personalised medicine.

\section{Introduction}

The hereditary spastic paraplegias (HSPs) are a heterogeneous group of monogenic neurological diseases with a combined prevalence of two to five cases per 100000 individuals worldwide. ${ }^{1,2}$ They are characterised by length-dependent corticospinal tract and dorsal column degeneration that manifests with the core clinical features of bilateral lower limb spasticity, hyperreflexia, and extensor plantar responses. ${ }^{3}$ HSPs can present in infancy, childhood, adolescence, or adulthood. Autosomal dominant, autosomal recessive, or X-linked modes of inheritance are reported, with $13-40 \%$ of cases being sporadic (ie, with no family history). ${ }^{4,5}$

The genetic classification for HSP is based on sequential numbering of chromosomal loci or specific genes, as they were identified, using a spastic paraplegia gene (SPG) designation. Up to 79 SPG genes have been described so far. Many of these have only been identified in single families, and so are perhaps best regarded as HSP candidate genes. ${ }^{6}$ Several other groups of monogenic neurological diseases, other than HSP, are associated with spasticity, usually in the context of other cardinal clinical features, and are not the focus of this Review. Despite the selective use of next-generation sequencingbased HSP gene panels or whole-exome sequencing, a genetic diagnosis is not made in $51-71 \%$ of all suspected cases of HSP, with or without a positive family history. ${ }^{4,5,7,8}$

This Review focuses on the more common HSPs encountered in clinical practice, highlighting clinical, neuroimaging, and neurophysiological features. We describe how to approach the diagnosis of a suspected case of HSP in the era of next-generation sequencing and propose a simple diagnostic algorithm using clinical and neuroimaging features. We then discuss symptomatic treatments and the potential emerging therapeutic options for specific causes of HSP based on their underlying molecular mechanisms.

\section{Clinical characteristics}

Most cases present with a slowly progressive gait disturbance of insidious onset. Onset in early childhood can manifest with delayed motor milestones and might initially be misdiagnosed as cerebral palsy. The spasticity, which can be slightly asymmetrical, occurs in the absence of limb weakness and is only demonstrable on walking. Asymptomatic upper limb hyperreflexia without spasticity is common and a brisk jaw jerk can occasionally be seen. ${ }^{8}$ Urinary symptoms related to detrusor instability or detrusor sphincter dyssynergia are frequent, usually occurring later in the disease course. ${ }^{9}$ Asymptomatic, or mildly symptomatic, impairment of vibration sensation caused by dorsal column degeneration is also common, whereas central pathways conveying other sensory modalities are less often involved. ${ }^{4}$

Additional neurological features such as cognitive impairment, ataxia, dysarthria, neuropathy, or seizures are seen in more than half of cases and can be the presenting feature. ${ }^{4}$ The clinical classification proposed by Harding ${ }^{10}$ divides HSP cases on this basis into pure or complicated, later revised to pure or complex. These specific clinical features are sometimes helpful for differentiating the underlying cause; although substantial phenotypic variation can occur between individuals with the same SPG type or specific mutation, and some features can be subtle The association between various different clinical features and the majority of SPG types, up to SPG78, has been reviewed before. ${ }^{12}$ Here, we cover the more common HSPs encountered in clinical practice divided according to inheritance patterns - given that a positive family history can immediately narrow the differential diagnosis.

\section{Autosomal-dominant HSP subtypes}

The SPG4 subtype is the most common HSP. This subtype is associated with mutations in SPAST and is inherited as an autosomal dominant trait. ${ }^{13-15}$ It accounts for up to a third of all HSP cases, including $60 \%$ of autosomal dominant cases and $15 \%$ of sporadic cases. ${ }^{4}$ The mean age of onset is 31.7 years but cases have been reported with onset of up to 70 years, and it usually presents with isolated lower limb spasticity, with or without bladder or sensory dysfunction. ${ }^{8}$ In a cohort of 196 patients of mostly German origin, ataxia or peripheral motor involvement were seen in $5-10 \%$ of cases and cognitive involvement, or subclinical, or only emerge later in the disease course.,11
Lancet Neurol 2019 Published Online July 31, 2019 http://dx.doi.org/10.1016/ S1474-4422(19)30235-2

Reta Lila Weston Institute of Neurological Studies (S Shribman MA Prof TTWarner PhD), and Department of Neuromuscular Diseases (Prof H Houlden PhD), UCL Queen Square Institute of Neurology, London, UK; Cambridge Institute for Medical Research and Department of Medical Genetics, University of Cambridge, Cambridge, UK (E Reid PhD); and The Institute of Biomedical and Clinical Science, University of Exeter Medical School, Exeter, UK (Prof A H Crosby PhD

Correspondence to Prof Thomas T Warner, Reta Lila Weston Institute of Neurological Studies, UCL Queen Square Institute of Neurology, London, WC1N 1PJ, UK t.warner@ucl.ac.uk 


\begin{tabular}{|c|c|}
\hline & Investigations \\
\hline \multicolumn{2}{|l|}{ Structural } \\
\hline Cervical spondylosis & MRI \\
\hline Neoplasia (spinal or parasagittal) & MRI \\
\hline Chiari malformation & MRI \\
\hline Atlanto-axial subluxation & MRI \\
\hline Tethered cord ${ }^{37}$ & MRI \\
\hline \multicolumn{2}{|l|}{ Vascular } \\
\hline Dural arteriovenous malformation & MRI, angiography \\
\hline \multicolumn{2}{|l|}{ Inflammatory } \\
\hline Primary progressive multiple sclerosis & MRI, CSF \\
\hline \multicolumn{2}{|l|}{ Infectious } \\
\hline HTLV1 & MRI, CSF, HTLV1 serology \\
\hline HIV & MRI, CSF, HIV serology \\
\hline Neurosyphilis & MRI, CSF, syphilis serology \\
\hline \multicolumn{2}{|l|}{ Metabolic } \\
\hline Vitamin B12 deficiency & $\begin{array}{l}\text { MRI, vitamin B12, methylmalonic } \\
\text { acid and homocysteine } \\
\text { concentrations }\end{array}$ \\
\hline Copper deficiency & MRI, serum copper \\
\hline \multicolumn{2}{|l|}{ Toxic } \\
\hline Lathyrism & History \\
\hline \multicolumn{2}{|l|}{ Neurodegenerative } \\
\hline Primary lateral sclerosis ${ }^{38}$ & MRI, electromyogram \\
\hline \multicolumn{2}{|l|}{ latrogenic } \\
\hline Radiation myelopathy ${ }^{39}$ & MRI, history \\
\hline \multicolumn{2}{|c|}{ Table adopted from Salinas and colleagues ${ }^{3}$. HTLV1=human T-lymphocytic virus 1.} \\
\hline $\begin{array}{l}\text { Table 1: Acquired causes of slowly pro } \\
\text { diagnostic investigations }\end{array}$ & gressive spastic paraplegia and ke \\
\hline
\end{tabular}

extrapyramidal involvement, dysarthria, or dysphagia were seen in fewer than $5 \%$ of cases. ${ }^{4}$

The SPG3A subtype is the second most common autosomal dominant HSP. This subtype is associated with mutations in ATL1 and constitutes around 5-10\% of autosomal dominant HSP cases that test negative for SPAST mutations. ${ }^{8}$ Similarly, it usually presents with isolated lower limb spasticity, with or without bladder or sensory dysfunction; however, an axonal motor neuropathy is seen in up to $25 \%$ of cases. ${ }^{4}$ The mean age of onset for SPG3A is considerably lower than SPG4 ( 5.6 years $v$ s 31.7 years), meaning that ATL1 and SPAST mutations occur with roughly equal frequency in autosomal dominant HSP presenting in the first decade of life, whereas autosomal dominant HSP presenting after the fourth decade is unlikely to be caused by ATL1. ${ }^{4}$

The SPG 31 subtype is the third most common autosomal dominant HSP and is associated with mutations in REEP1. ${ }^{16,17}$ Like SPG3A, it usually presents as a pure phenotype, with or without bladder or sensory dysfunction, but is associated with an axonal neuropathy in up to $50 \%$ of cases..$^{18}$ It appears to have a bimodal distribution with peaks in the first and fourth decades, although onset in a nonagenarian has been reported, and the age of onset can also vary greatly within individual families. ${ }^{19}$
Several patients with SPG31 have been reported to have COX-deficient fibres on muscle biopsy, which suggests mitochondrial dysfunction. ${ }^{18}$

\section{Autosomal recessive HSP subtypes}

The SPG11 subtype is one of the most prevalent autosomal recessive HSPs. It is associated with mutations in SPG11 and accounts for up to $8 \%$ of all HSP cases, with a higher prevalence in populations with considerable consanguinity. ${ }^{1,5}$ It presents between the ages of 4 years and 36 years. The phenotype can vary between family members but is invariably complex. Most cases present with cognitive impairment or learning difficulties with lower limb spasticity emerging later, typically in the second decade. ${ }^{20}$ More than $50 \%$ of patients develop dysarthria, ataxia, axonal motor neuropathy, or prominent urinary symptoms. ${ }^{4,20}$ Progression to upper limb involvement is common and levodopa-responsive parkinsonism, oromandibular dystonia, seizures, and visual failure secondary to optic atrophy have also been reported.?

The SPG15 subtype is associated with mutations in ZFYVE26. This subtype presents in a similar way to SPG11; although the axonal neuropathy can be more prominent and levodopa-responsive parkinsonism is more frequent. ${ }^{20}$ It can also cause adducted thumbs, an unusual sign seen in cases of SPG1, an X-linked HSP also known as mental retardation, aphasia, shuffling gait, and adducted thumbs (MASA) syndrome., ${ }^{7,21}$

The SPG7 subtype is associated with mutations in SPG7. It presents later than other HSPs, with a mean age of onset of $41 \cdot 7$ years, ${ }^{4,22}$ and appears to be more common in men than in women. ${ }^{23}$ It usually manifests with a combination of lower limb spasticity, which can be relatively mild, and cerebellar ataxia. ${ }^{24}$ Cerebellar signs were seen in $57-90 \%$ of people and dysarthria in $37-76 \%$ of individuals at presentation in the two largest cohorts of 42 patients from England and 49 patients from the Netherlands. ${ }^{23,25}$ A waddling gait, associated with proximal myopathic weakness, is also common, and progressive external ophthalmoplegia is also seen, and can be a useful diagnostic clue. ${ }^{26}$ These features reflect underlying mitochondrial dysfunction that can be confirmed on muscle biopsy. ${ }^{27,28}$ Visual loss due to optic neuropathy is a rare presentation of SPG7, ${ }^{25}$ but subclinical abnormalities in ocular coherence tomography were identified in all cases from one French cohort of ten patients. ${ }^{22}$ This HSP was previously considered to be rare and exclusively autosomal recessive; ${ }^{29}$ however, the carrier frequency of the Ala510Val mutation, which is suspected to be pathogenic, was predicted to be as high as $3-4 \%$ in the UK population. ${ }^{30-32}$ Such high carrier frequency raises the possibility of reduced penetrance in individuals who are homozygous for this mutation, thought to act as a hypomorphic allele. Autosomal dominant inheritance has also been reported in several families.22 In at least one case this inheritance was related to a segregating third allele (ie, pseudodominance), although, some relatives with a 
single heterozygous Ala5210Val mutation also developed a late onset and relatively mild cerebellar ataxia. However, it is possible that an alternative genetic diagnosis was present. ${ }^{22,25,33}$ Perhaps some caution is therefore advised when providing genetic counselling, but in view of the high prevalence of Ala510Val, the heterozygous carriers of the mutation are unlikely to be at a substantially increased risk of a neurological condition.

SPG5 is associated with mutations in CYP7B1. It is an autosomal recessive HSP that is relatively rare in most cohorts, but made up 28 of 101 autosomal recessive cases in a large Chinese study of 531 patients. . $5,7,34,35^{\text {An inter- }}$ national cohort of 34, predominantly European, patients was described by Schols and colleagues. ${ }^{36}$ The median age of onset was 13 years (range 1-33). Dorsal column dysfunction was seen in 32 patients (94\%), in whom it was unusually severe, and in 16 patients (47\%), in whom the presentation was a sensory ataxia in the lower limbs. Urinary symptoms were also prominent and five patients (15\%) also reported rectal urgency or incontinence. Cognitive impairment does not appear to be a prominent feature. ${ }^{4,36}$

\section{Diagnosis}

The identification of pathogenic mutations in an SPGdesignated gene is required to confirm a molecular diagnosis of HSP. However, the clinical priority should always be to exclude acquired causes for progressive spastic paraparesis in the first instance. These include a range of structural, inflammatory, and infectious causes, in addition to amyotrophic or primary lateral sclerosis, arteriovenous fistulas, vitamin B12 deficiency, and copper deficiency (table 1).,37-39 Assessment of very long chain fatty acids should be considered in any family with no documented male-to-male transmission of the disease to exclude adrenoleukodystrophy. A detailed developmental and family history can provide important clues to a diagnosis of HSP whereas acute or subacute deterioration suggests acquired causes.

\section{Neuroimaging}

MRI of the spinal cord is unremarkable or shows thinning of the spinal cord for most SPG types, ${ }^{40}$ with the notable exception of SPG2 (associated with mutations in PLP1), a rare X-linked HSP and variant of PelizaeusMerzbacher disease, which can be associated with a diffuse pattern of hypomyelination. ${ }^{41} \mathrm{~T} 2$-weighted hyperintensities in the spinal cord should prompt careful reconsideration of acquired causes. Other rare genetic and metabolic causes associated with T2-weighted abnormalities in the spinal cord have been reviewed by Marelli and colleagues. ${ }^{42}$

MRI of the brain can help differentiate SPG types, although only a few pathognomonic changes are known. Cerebellar atrophy is seen in 39-95\% of SPG7 cases, mostly as mild atrophy of the cerebellar vermis (figure 1):23,25 however, this atrophy has also been reported in other rarer
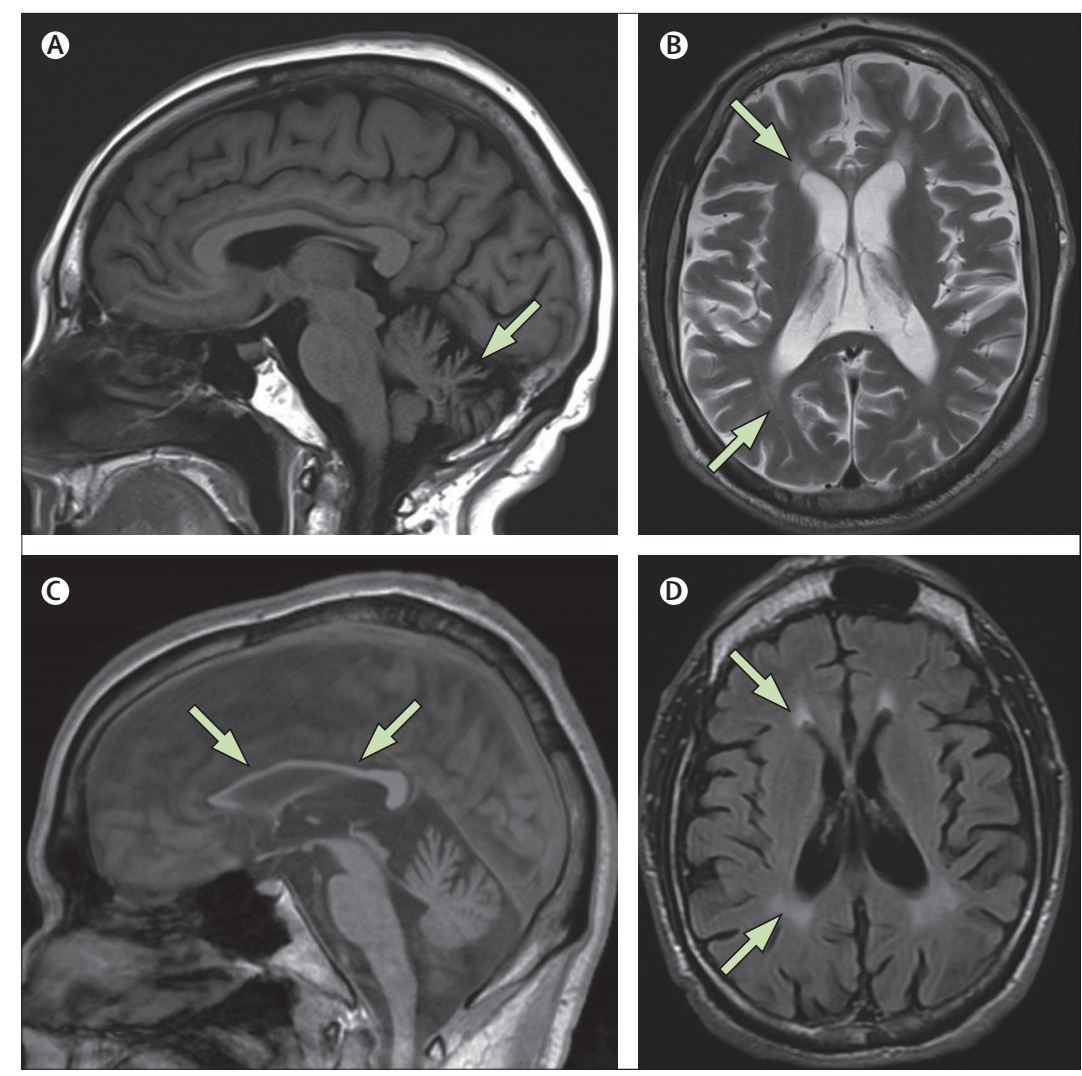

Figure 1: Brain imaging in patients with SPG7 and SPG15

(A) A T1 sagittal image showing cerebellar atrophy in a patient with SPG7 (B) A T2 axial image and (D) a T2 axial image with fluid attenuated inversion recovery known as FLAIR, which both show white matter hyperintensities in a patient with SPG15. (C) A T1 sagittal image showing a thin corpus callosum in the same patient with SPG15 White matter hyperintensities and a thin corpus callosum are also seen in SPG11 HSP. HSP=hereditary spastic paraplegia.

HSPs. The dentate nucleus, which is usually hypointense on T2-weighted imaging, appeared isointense or hyperintense relative to pontine white matter in $86 \%$ of cases in a series of 42 patients with SPG7, and might be a more specific finding. ${ }^{23}$ Thinning of the corpus callosum and periventricular white matter hyperintensities are seen in nearly all cases of SPG11 and SP15 (figure 1), and in other rarer subtypes of complex HSP. ${ }^{20}$ The so-called ears of the lynx sign, corresponding to a specific pattern of T2-weighted hyperintensities at the anterior forceps of the corpus callosum, is associated with SPG11 and SPG15. ${ }^{43}$ Non-specific white matter lesions are also seen in up to $50 \%$ of complex SPG 5 cases. ${ }^{34}$ We recommend interpretation by a neuroradiologist with expertise in leukodystrophy if white matter abnormalities of uncertain significance are identified.

\section{Neurophysiology}

Neurophysiological findings were systematically studied in an Italian cohort of 70 patients with HSP (the majority with SPG4 and SPG11) by Martinuzzi and colleagues. ${ }^{44}$ Central motor conduction times were absent or delayed in the lower limbs in 31 of 32 patients. The patient with 


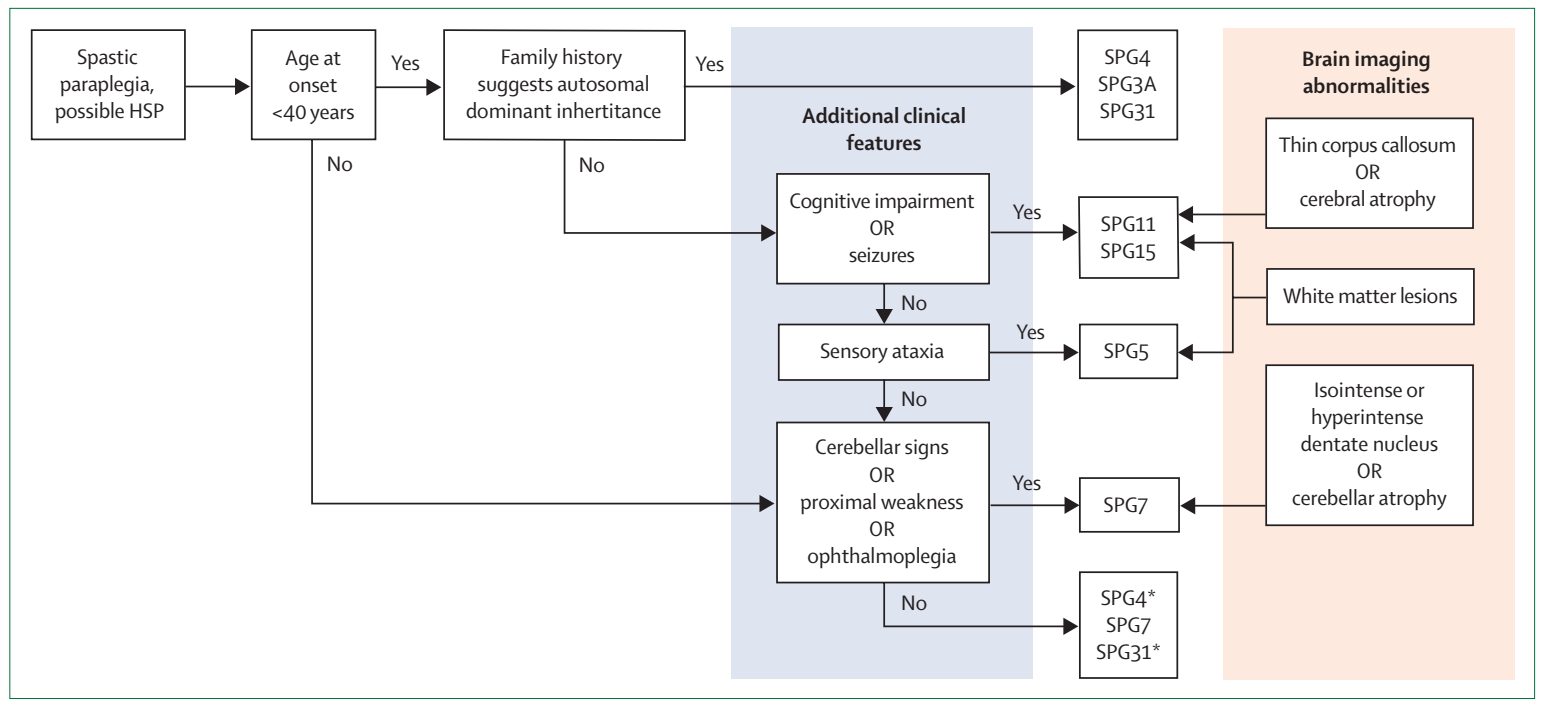

Figure 2: Proposed diagnostic algorithm for common hereditary spastic paraplegias

The purpose of this proposed algorithm is to guide clinicians who are presented with potential cases of hereditary spastic paraplegia, and combines the inheritance pattern and additional clinical features with associated brain imaging abnormalities at presentation. It may be of value where only limited genetic testing is available to guide which genotypes to consider. It is important to note that this algorithm has not been tested prospectively. HSP=hereditary spastic paraplegia. *de novo mutation or insufficient family information.

normal central motor conduction times had a SPAST mutation with a very mild phenotype. Central motor conduction times were abnormal in the upper limbs in 14 of the 31 patients with abnormal lower limb studies. Somatosensory evoked potentials were abnormal in 30 of 44 patients. Electromyography and nerve conductions studies identified distal axonal motor neuropathy and associated neurogenic changes in 23 of 49 patients with HSP. These included most SPG7, SPG11, and SPG15 cases and some SPG3A, SPG4, and SPG5 cases. A neurophysiological study of the SPG31 subtype of HSP identified at least one focal mononeuropathy in all eight patients, including features of carpal tunnel syndrome in seven patients (13 of 16 hands), and one case of multifocal compression neuropathy, suggesting a peripheral predisposition to pressure palsies. ${ }^{45}$ Abnormalities in neurophysiological testing are therefore common in HSP but do not seem to be specific to subtypes, with the exception of focal mononeuropathy, particularly of the upper limb, which appears to be common in SPG31 cases.

\section{Genetic testing}

Several different approaches to genetic testing for HSP can be used. ${ }^{16,46}$ Next-generation sequencing-based gene panels for HSP are increasingly cost-efficient and now widely available. These panels are most commonly used to screen the exons of a large number of genes associated with HSP but have their limitations; they will generally not identify copy number variants (ie, large deletions or duplications, including exon deletions), mutations in promotor or deep intronic regions, and triplet repeat disorders. ${ }^{47}$ This technical limitation is a particular problem for SPAST, where exon deletions are relatively common. Multiplex ligation-dependent probe amplification is recommended for this gene if sequencing approaches give normal results. ${ }^{48}$ Some centres currently use first-generation sequencing methods in a small number of targeted genes for initial genetic testing and consider next-generation sequencing-based gene panels if results are inconclusive. We believe that our proposed diagnostic algorithm in figure 2 can be particularly helpful in this scenario but it is important to note that it has not been tested prospectively in this context. This algorithm may also be helpful at centres that prefer to perform panel sequencing as a first-line of investigation; either when no pathogenic mutations are identified and the clinician needs to decide which genes should be tested with multiple ligation-dependent probe amplification, or, when a variant of uncertain significance is identified and correlation with phenotype is required.

Clinicians also need to be aware that several other groups of monogenic diseases can present with slowly progressive lower limb spasticity without spinal cord imaging abnormalities and are not represented in the SPG classification. They might not be comprehensively covered by some next-generation sequencing panels and include the spinocerebellar ataxias, autosomal recessive ataxias, spastic ataxias, demyelinating and hypomyelinating leukodystrophies, and other rare metabolic and neurodegenerative disorders (table 2) ${ }^{49-56}$ Several of the diseases in these groups, such as spinocerebellar ataxias type 1 (ATXN1) and type 3 (ATXN3) and Friedreich's ataxia $(F X N)$, are triplet repeat disorders and might not be covered by gene panels, even if they are extended to include wider groups of monogenic diseases associated with spasticity. ${ }^{57-59}$ 
Particular attention should be given to atypical presentations of treatable diseases in which lower limb spasticity occurs, often in the context of other clinical features, in the absence of T2-weighted imaging abnormalities in the spinal cord. These presentations include adrenoleukodystophy (caused by mutations in $A B C D 1$ ), arginase deficiency (ARG1) ${ }^{61}$ cerebrotendinous xanthomatosis (CYP27A1) ${ }^{62}$ dopa-responsive dystonia (GCH1, TH, and other genes) ${ }^{63}$ phenylketonuria $(P A H),{ }^{64}$ biotinidase deficiency $(B T D),{ }^{65}$ cobalamin-related remethylation disorders, ${ }^{66}$ methylenetetrahydrofolate reductase deficiency $($ MTHFR $),{ }^{67}$ and primary coenzyme Q10 deficiencies. ${ }^{68}$

\section{Disease progression and prognosis}

Data for prognosis for individual SPG types are scarce except that SPG11 is associated with a higher disease severity, as determined by the Spastic Paraparesis Rating Scale, relative to other HSPs. ${ }^{4}$ Disease progression in HSPs overall is usually slow although a later onset is associated with earlier loss of independent walking. The average rate of disease progression was observed to be slower for patients with SPG3A than SPG4 in one cohort, but did not differ when comparing individuals with onset before 20 years. $^{8}$ In the largest retrospective cohort, the median disease duration until loss of independent walking was 22 years. ${ }^{4}$ This German cohort of 608 patients included 198 (33\%) SPG4, 28 (5\%) SPG7, and 15 (2\%) SPG11 cases, among numerous rarer SPG types. After a disease duration of 20 years, $48 \%$ used a walking aid and $12 \%$ used a wheelchair. After a disease duration of 40 years, $72 \%$ used a walking aid and $29 \%$ used a wheelchair. ${ }^{4}$

\section{Management of HSP \\ Symptomatic treatment}

The ideal approach for the management of symptoms is in a multidisciplinary spasticity clinic where the stiffness, cramps, spasms, and deformities can be addressed. An exercise programme supervised by neurophysiotherapists, focused initially on stretches, and later balance, is optimal. Use of orthotics, such as ankle-foot orthoses and heel raises, is also valuable for mobility.

Oral antispasmodics, including baclofen and tizanidine, have an established role, although most patients do not gain significant clinical benefit. Gabapentin has been used for spasticity in multiple sclerosis and spinal cord injury, but a double blind crossover trial in a small cohort of SPG4 cases found no difference in effect from placebo. ${ }^{69}$ An open-label trial of fampridine (4-aminopyridine) in a mixed cohort of HSP (SPG4, SPG7, SPG11) over 2 weeks suggested some benefit and deserves more rigorous testing. ${ }^{70}$ With more severe spasticity intrathecal baclofen can be effective in reducing very high tone associated with pain and disability. It is often used for patients requiring wheelchairs, but also has been shown to improve gait if used earlier. ${ }^{71}$

Botulinum toxin injections can be helpful for targeting specific problematic muscle groups around the ankle, knee, and hip. One trial enrolled patients with SPG4, SPG3A, and SPG8 and studied the combination of injections into the calf and stretching exercises, showing that this combined treatment improved gait velocity and reduced muscle tone, while preserving strength and balance. $^{72}$

\begin{tabular}{|c|c|c|}
\hline & Gene & $\begin{array}{l}\text { Investigations (excluding } \\
\text { gene tests) }\end{array}$ \\
\hline \multicolumn{3}{|l|}{ Leukodystrophies } \\
\hline Adrenoleukodystrophy* & $A B C D 1$ & Very long chain fatty acids \\
\hline Aicardi-Goutieres syndrome & $\begin{array}{l}\text { IFIH1, ADAR, KLC2, } \\
\text { RNASEH2B }\end{array}$ &.. \\
\hline $\begin{array}{l}\text { Autosomal dominant adult-onset demyelinating } \\
\text { leukodystrophy }\end{array}$ & LMNB1 & .. \\
\hline Adult polyglucosan body diseases* & GBE1 & .. \\
\hline Alexander disease & GFAP & .. \\
\hline Cerebrotendinous xanthomatosis* & CYP27A1 & Cholestanol \\
\hline $\begin{array}{l}\text { Hypomyelinating leukodystrophy with atrophy of } \\
\text { basal ganglia and cerebellum }\end{array}$ & TUBB4A & \\
\hline $\begin{array}{l}\text { Hypomyelination with brainstem and spinal } \\
\text { involvement and leg spasticity }\end{array}$ & DARS &.$\cdot$ \\
\hline $\begin{array}{l}\text { Tay-Sachs disease, Sandhoffs disease, and GM2 } \\
\text { activator deficiency }\end{array}$ & HEXA, HEXB, GM2A & $\begin{array}{l}\text { Hexosaminidase } A \text {, total } \\
\text { beta-hexosaminadase }\end{array}$ \\
\hline Globoid cell leukodystrophy (Krabbe's disease) & GALC & Galactocerebrosidase \\
\hline $\begin{array}{l}\text { Leukoencephalopathy with brainstem, spinal cord } \\
\text { involvement, and lactate elevation }\end{array}$ & DARS2 & .. \\
\hline Metachromatic leukodystrophy & ARSA & Arylsulfatase A \\
\hline Pelizaeus-Merzbacher disease & PLP1 & .. \\
\hline Pelizaeus-Merzbacher-like disease & GJC2 & .. \\
\hline \multicolumn{3}{|l|}{ Other metabolic disorders } \\
\hline Arginase deficiency* & ARG1 & Plasma amino acids \\
\hline Biotinidase deficiency ${ }^{*}$ & BTD & Biotinidase \\
\hline $\begin{array}{l}\text { Cobalamin-related remethylation disorders (cblC, } \\
\text { cblD, cblE, cblF, cblG, cblH, cbl), and } \\
\text { methylenetetrahydrofolate reductase deficiency) }\end{array}$ & $\begin{array}{l}\text { MMACHC, MMADHC, } \\
\text { LMBRD1, ABCD4, } \\
\text { MMADHC, MTRR, MTR, } \\
\text { MTHFR }\end{array}$ & Homocysteine \\
\hline Glucose transporter type 1 deficiency syndrome & SLC2A1 & CSF glucose \\
\hline Dopa-responsive dystonia & GCH, TH, FTPS,SPR & $\begin{array}{l}\text { Levodopa trial, CSF pterins, } \\
\text { and CSF neurotransmitters }\end{array}$ \\
\hline Phenylketonuria* & PAH & Plasma amino acids \\
\hline Primary coenzyme Q10 deficiencies* & $\begin{array}{l}\text { COQ2, APTX, PDSS1, } \\
\text { PDSS2, CABC1, COQ9 }\end{array}$ &.. \\
\hline \multicolumn{3}{|l|}{ Spinocerebellar ataxias } \\
\hline Type 1 & ATXN1 & .. \\
\hline Type 3 (Machado-Joseph disease) & ATXN3 & .. \\
\hline \multicolumn{3}{|l|}{ Autosomal-recessive ataxias } \\
\hline $\begin{array}{l}\text { Autosomal-recessive spastic ataxia of } \\
\text { Charlevoix-Saguenay }\end{array}$ & SACS & Nerve conduction studies \\
\hline Friedreich's ataxia & FXN & Nerve conduction studies \\
\hline \multicolumn{3}{|l|}{ Spastic ataxias } \\
\hline Type 1 & VAMP1 &.. \\
\hline Type 2 & KIF1C & .. \\
\hline Type 3 & MARS2 &.$\cdot$ \\
\hline Type 4 & MTPAP & .. \\
\hline Type 5 & AFG3L2 & .. \\
\hline
\end{tabular}

(Table 2 continues on next page) 


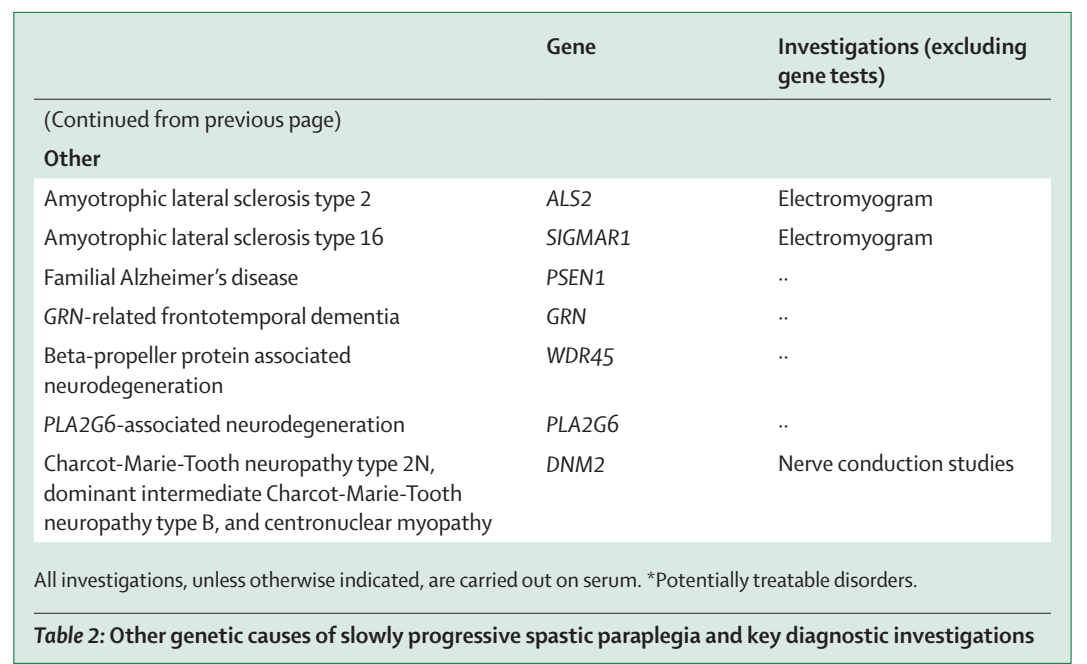

Functional electrical stimulation might improve gait disturbance in patients with HSP. In a small crossover study of 11 UK patients with spastic paraparesis, including six participants with a positive family history, bilateral common peroneal stimulation or another preferred pattern of stimulation (hip abductors, lumbar extensor muscles, or the flexor withdrawal reflex), improved toe clearance and dorsiflexion in the swing phase and significantly improved walking speed. ${ }^{73}$ The use of transcutaneous direct current spinal stimulation has also been shown to have a positive effect on spasticity in a crossover study with 11 patients with HSP from Italy, although the stimulation did not affect a walking test. ${ }^{74}$

The value of the multidisciplinary approach to patients with HSP was shown in a 2017 publication that found that detailed clinical information and profiling, associated with combined therapies with oral medication and botulinum toxin plus physiotherapy improved patients' gait, and reduced falls. ${ }^{75}$

The complexity of the problems with gait has been highlighted by a study of 29 patients with HSP (mainly SPG4, but also SPG3A, SPG5, SPG7, and SPG31) and 30 controls with kinematics and electromyography of 12 leg muscles while walking. The findings showed that degeneration of corticospinal tracts in HSP was associated with widening of spinal locomotor output to muscles spreading from caudal to rostral segments, starting in the sacral region. ${ }^{76}$ This evolving process can be one reason why antispasmodic medication is not as efficacious in treating patients with HSP as it is in patients with an acute inflammatory or traumatic spinal event. Treating patients with HSP might require a more targeted approach, such as retraining other pathways including the reticulospinal tract, ${ }^{\pi}$ or robotic gait training, which has shown benefit to both balance and walking. ${ }^{78}$

Although most attention has focused on managing the spasticity and gait, little consideration of other symptoms has been seen, especially urinary symptoms. A study of 71 patients with HSP from Germany, including 34 SPG4 cases, highlighted this need using validated rating scales. One or more urinary symptoms were seen in 53 patients (75\%), incontinence was higher in women and SPAST mutation carriers associated with worse symptoms. ${ }^{79}$ Over a third of patients had received no treatment for their urinary problems. These urinary problems can often be considerably improved by oxybutynin or equivalent drugs, after exclusion of urinary tract infection and ensuring that there is not considerably increased postmicturition residual volume of urine.

\section{Genotype-specific therapies}

The prominent genetic and clinical heterogeneity of HSP reflects a variety of pathogenic mechanisms that underlie the slow terminal axonal degeneration. Although these processes can eventually resolve into a few pathogenic pathways, at present the cellular processes implicated in the axonopathy include membrane trafficking, mitochondrial function, organelle biogenesis and shaping, axonal transport, and lipid and cholesterol metabolism, and these have been comprehensively reviewed. ${ }^{12}$

Although many genetic neurological disorders, such as Huntington's disease and spinal muscular atrophy, have seen substantial advances in bespoke genotype-targeted therapy, the same is not true for HSP. This lack of progress relates to the relative rarity of specific HSP genetic subtypes, extensive genetic heterogeneity and consequent mechanistic diversity, and slowly progressive clinical course of most cases. However, the research to investigate specific treatments for genetic HSP subtypes has been increasing, including treatments attempting to address pathophysiological mechanisms and gene therapy. The following sections summarise the molecular pathogenesis and potential therapeutic targets for the common HSP subtypes highlighted in this Review.

\section{Mechanistic approaches to therapy \\ SPG4}

The SPAST gene encodes spastin, a member of the ATPases associated with diverse cellular activities protein family. Spastin is involved in microtubule severing and regulation of microtubule dynamics. ${ }^{80}$ Reduced concentrations of spastin in cell models are associated with lower numbers of dynamic microtubules and increased stable acetylated microtubules. ${ }^{81}$ Spastin is believed to control a range of microtubule-dependent processes including axonal transport, endosomal recycling, lysosomal function, cytokinesis, and endoplasmic reticulum shaping. It has two cellular isoforms, the full length M1 isoform and the smaller M87 isoform, generated by a complex transcriptional mechanism. All forms of spastin possess a microtubule interacting and trafficking (MIT) domain that binds endosomal sorting complexes required for transport (ESCRT) proteins, which have a role in the recognition and sorting of ubiquitin-conjugated proteins into internal vesicles of the late endosome, a crucial process for receptor degradation in lysosomes. ${ }^{82}$ The MIT 
domain is also involved in recruitment of spastin to endosomes and, during cell division, cytokinetic midbodies. Additionally, spastin regulates axonal and synaptic growth, and neurite branching, ${ }^{83,84}$ and recently spastin variants have been implicated in defective bone morphogenetic protein (BMP) and neuropilin-1 signalling affecting motor axon navigation. ${ }^{85}$ Finally, spastin is involved in regulating axonal transport of receptors, vesicles and organelles including mitochondria, which can be found in axonal swelling in models of the SPG4 subtype of HSP. ${ }^{86}$

An unbiased approach with null SPAST homologues in Caenorhabditis elegans, drosophila, and zebrafish to identify drugs that could rescue the HSP phenotype, screening with compounds known to modulate endoplasmic reticulum (ER) stress, has been done. ${ }^{87}$ Locomotor defects and lifespan in all three models could be partly rescued by phenazine, methylene blue, $N$-acetyl-cysteine, guanabenz, and salubrinal, and markers of ER stress levels correlated with improved locomotor activity. Future work is needed in mammalian models to assess clinical relevance, but the use of compounds approved by Food and Drug Administration (US FDA) in this study holds the promise of rapid translation to human therapy if further work yields positive results.

The role of spastin in microtubule stability has also been investigated in drosophila and in neurons derived from human-induced pluripotent and olfactory stem cells. This work showed that microtubule destabilising drugs, including vinblastine, attenuated the mutant spastin-associated phenotype, showing the potential for mechanistic targeting. ${ }^{88,89}$ Furthermore, use of vinblastine and nocodazole rescued focal axonal swellings associated with impaired axonal transport in cortical neurons from spastin knockout mice. ${ }^{90}$

The SPG4 subtype of HSP is, in most cases, widely believed to be mediated by haploinsufficiency and partial loss of function in microtubule severing. If true, this opens the possibility of gene therapy to rescue the loss of function. One study in neurons derived from human induced pluripotent stem cells from a patient with a SPAST nonsense mutation found that overexpression of the M1 or M87 spastin isoforms restored neurite length, branching, and numbers of primary neurites, and reduced swellings in neuronal cells. ${ }^{91}$ However, this work has not been pursued further in animal models. This approach might not be the best therapeutic strategy in the case of a subset of SPAST missense mutations, where dominant-negative or, perhaps less likely, gain-of-function mechanisms might contribute to their pathogenesis. ${ }^{13}$

\section{SPG5}

Potentially, one of the most treatable forms of HSP is the SPG5 subtype. This autosomal recessive form of HSP is caused by mutations in the CYP7B1 gene encoding oxysterol-7 $\alpha$-hydroxylase, which is involved in the degradation of cholesterol into primary bile acids and leads to accumulation of neurotoxic oxysterols. ${ }^{36}$ The metabolic defect can be detected by increased concentrations of 25-hydroxycholesterol and 27-hydroxycholesterol in the plasma and CSF. Treatment with cholesterol-lowering drugs (ie, atorvastatin) reduced the concentration of plasma 27-hydroxycholesterol. ${ }^{36,92}$ Another study also detected abnormal bile acids profile in patients with SPG5, which could be corrected by chenodeoxycholic acid, and suggested that combination therapy with atorvastatin and chenodeoxycholic acid might be beneficial..$^{93}$ Long term trials are required to determine whether these strategies improve clinical outcomes.

\section{SPG3A and SPG31}

ATL1 encodes atlastin-1, an integral membrane, ER-localised GTPase, enriched in the CNS, ${ }^{94}$ which is involved in the fusion of ER tubules to form polygonal ER network. Numerous HSP proteins localise to the tubular ER and interact with each other to help create and regulate the tubular ER network, including M1 spastin, atlastin-1, REEP-1, and others. Mutations in the genes encoding these proteins can disrupt correct ER morphology and have been proposed to cause axonopathy by altering the function of axonal ER. ${ }^{95-97}$ Perhaps related to these ER phenotypes, abnormalities in lipid droplets, which form at the ER, have been reported in REEP-1 null mice and atlastin models. ${ }^{98,99}$ Finally, a potential therapeutic effect of microtubule destabilising drugs (ie, vinblastine and taxol, produced by Sigma) was shown in rescue of axon growth defects in neurons derived from patient induced pluripotent stem cells (iPSC) with

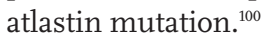

\section{SPG7}

The protein product of SPG7 is paraplegin, a metalloprotease of the inner mitochondrial membrane. Oxidative phosphorylation defects in muscle from patients have been demonstrated, and SPG7 null mice have mitochondrial-filled axonal swellings, which shows a link between transport and mitochondrial function. ${ }^{28,101}$ Paraplegin is also an essential component of the mitochondrial permeability transition pore, ${ }^{102}$ and a SPG7 variant that escapes normal processing leads to heightened ATP and reactive oxygen species production. This SPG7 variant is further associated with non-neurological conditions, such as type 2 diabetes and coronary artery disease, and toxicity of chemotherapeutic agents. ${ }^{103}$ These loss-of-function mitochondrial diseases can affect central and peripheral neurons, and one study with adeno-associated virusmediated intramuscular delivery of paraplegin in a mouse model halted progression of neuropathology and rescued mitochondrial morphology in peripheral nerves. ${ }^{104}$

\section{SPG11 and SPG15}

The clinical similarity between SPG11 and SPG15 subtypes of HSP reflects a common underlying pathogenetic mechanism. Both of the encoded proteins, spatacsin and 


\section{Search strategy and selection criteria}

We identified references for this Review by searches of PubMed between Aug 1, 2008, and Oct 1, 2018 and from relevant articles with no language restrictions. The search terms "hereditary spastic paraplegia", "hereditary spastic paraparesis", "SPG3", "SPG3A", "SPG4", "SPG7", "SPG11", "SPG15", and "SPG31" were used. We generated the final reference list on the basis of relevance to the topics covered in this Review.

spastizin, interact with the AP5 vesicle adapter complex..$^{105}$ They both affect cellular autophagic processes, in particular autophagic lysosome reformation, a pathway that generates new lysosomes in the cell. Spatacsin loss of function appears to produce lysosome depletion through abnormal lysosomal lipid clearance, ${ }^{106,107}$ which affects initiation of lysosomal tubulation. ${ }^{108}$ Loss of function of spastizin has been found to affect processes involving the intersection between endocytosis and autophagy. ${ }^{109}$

One study has attempted to target the pathogenesis of SPG11 subtype on the basis of work with iPSC-derived neural precursor cells. Reduced proliferation of neural precursor cells from SPG11 patients was found to be mediated by increased GSK3B activity leading to impaired $\beta$-catenin signaling. ${ }^{110}$ Pozner and colleagues ${ }^{111}$ tested the GSK3 $\beta$ inhibitor tideglusib on neuronal lines derived from iPSC of a patient with SPG11 and on gene-edited lines. This treatment rescued neuritic pathology in the cells and decreased cell death, suggesting tideglusib (or similar molecules) as a candidate for further translational work.

\section{Conclusions and future directions}

HSP is a phenotypically and genotypically diverse group of monogenic diseases with emerging opportunities to provide targeted molecular therapies and precision medicine. The advances in molecular biology over the past 20 years have benefitted diagnosis and gene identification for numerous HSP subtypes. The rapid increase in known causal genes has led to identification of a large number of potential mechanisms for axonal degeneration. This heterogeneity has meant identifying tractable generic therapeutic targets for HSP is challenging.

The field is also hampered by the fact that, despite being a disabling group of motor neuron diseases, in most cases progression is slow. A clinical trial of a diseasemodifying agent would be difficult to envisage for many forms as only a few biomarkers exist that would change over a reasonable period of time. Therefore, effort should be focused on exemplar HSP genotypes in which work could be used to identify biomarkers (rating scales, blood, and CSF) that are sensitive to change. One example is the Pre-SPG4 study in Germany looking at the longitudinal course and biomarkers in pre-symptomatic SPAST mutation carriers (NCT03206190).

Choosing subtypes amenable to therapy, such as SPG5, where a biochemical approach might benefit, are important. For developing gene therapy, the more common recessive forms, SPG11, SPG15, and SPG7, where loss of function is clear, also offer opportunities for targeted gene replacement or editing. International groups should pool resources into these areas and develop trialready cohorts to take advantage of progress in gene therapy or other therapeutic areas. Such efforts are beginning.

The following years will see the identification of yet more HSP loci. However, we also hope that they will bring the beginning of rationally designed therapeutic approaches for HSP.

\section{Contributors}

SS and TTW searched the literature and drafted the manuscript. SS designed the figures and tables with input from HH. All authors commented on and revised the final manuscript.

\section{Declaration of interests}

ER has received funding from Takeda for hereditary spastic paraplegiarelated research. All other authors declare no competing interests.

\section{Acknowledgments}

We wish to thank all the clinicians, scientists, and families that have contributed to research on HSP in the aforementioned studies.

\section{References}

1 Ruano L, Melo C, Silva MC, Coutinho P. The global epidemiology of hereditary ataxia and spastic paraplegia: a systematic review of prevalence studies. Neuroepidemiology 2014; 42: 174-83.

2 Coutinho P, Ruano L, Loureiro JL, et al. Hereditary ataxia and spastic paraplegia in Portugal: a population-based prevalence study. JAMA Neurol 2013; 70: 746-55.

3 Salinas S, Proukakis C, Crosby A, Warner TT. Hereditary spastic paraplegia: clinical features and pathogenetic mechanisms. Lancet Neurol 2008; 7: 1127-38.

4 Schule R, Wiethoff S, Martus P, et al. Hereditary spastic paraplegia: clinicogenetic lessons from 608 patients. Ann Neurol 2016; 79: 646-58.

5 Chrestian N, Dupre N, Gan-Or Z, et al. Clinical and genetic study of hereditary spastic paraplegia in Canada. Neurol Genet 2017; 3: e122.

6 Das Bhowmik A, Patil SJ, Deshpande DV, Bhat V, Dalal A. Novel splice-site variant of UCHL1 in an Indian family with autosomal recessive spastic paraplegia-79. J Hum Genet 2018; 63: 927-33.

7 Kara E, Tucci A, Manzoni C, et al. Genetic and phenotypic characterization of complex hereditary spastic paraplegia. Brain 2016; 139: 1904-18

8 Loureiro JL, Brandao E, Ruano L, et al. Autosomal dominant spastic paraplegias: a review of 89 families resulting from a portuguese survey. JAMA Neurol 2013; 70: 481-87.

9 Fourtassi M, Jacquin-Courtois S, Scheiber-Nogueira MC, et al. Bladder dysfunction in hereditary spastic paraplegia: a clinical and urodynamic evaluation. Spinal Cord 2012; 50: 558-62.

10 Harding AE. Classification of the hereditary ataxias and paraplegias. Lancet 1983; 321: 1151-55.

11 Fink JK. Hereditary spastic paraplegia: clinical principles and genetic advances. Semin Neurol 2014; 34: 293-305.

12 Blackstone C. Hereditary spastic paraplegia. Handb Clin Neurol 2018; 148: 633-52.

13 Solowska JM, Baas PW. Hereditary spastic paraplegia SPG4: what is known and not known about the disease. Brain 2015; 138: 2471-84.

14 Balicza P, Grosz Z, Gonzalez MA, et al. Genetic background of the hereditary spastic paraplegia phenotypes in Hungary - An analysis of 58 probands. J Neurol Sci 2016; 364: 116-21.

15 Proukakis C, Auer-Grumbach M, Wagner K, et al. Screening of patients with hereditary spastic paraplegia reveals seven novel mutations in the SPG4 (Spastin) gene. Hum Mutat 2003; 21: 70 .

16 Koh K, Ishiura H, Tsuji S, Takiyama Y. JASPAC: Japan Spastic Paraplegia Research Consortium. Brain Sci 2018; 8: 153. 
17 Zuchner S, Wang G, Tran-Viet KN, et al. Mutations in the novel mitochondrial protein REEP1 cause hereditary spastic paraplegia type 31. Am J Hum Genet 2006; 79: 365-69.

18 Goizet C, Depienne C, Benard G, et al. REEP1 mutations in SPG31: frequency, mutational spectrum, and potential association with mitochondrial morpho-functional dysfunction. Hum Mutat 2011; 32: 1118-27.

19 Beetz C, Schule R, Deconinck T, et al. REEP1 mutation spectrum and genotype/phenotype correlation in hereditary spastic paraplegia type 31. Brain 2008; 131: 1078-86.

20 Pensato V, Castellotti B, Gellera C, et al. Overlapping phenotypes in complex spastic paraplegias SPG11, SPG15, SPG35 and SPG48. Brain 2014; 137: 1907-20.

21 Schrander-Stumpel C, Howeler C, Jones M, et al. Spectrum of X-linked hydrocephalus (HSAS), MASA syndrome, and complicated spastic paraplegia (SPG1): clinical review with six additional families. Am H Med Genet 1995; 57: 107-16.

22 Klebe S, Depienne C, Gerber S, et al. Spastic paraplegia gene 7 in patients with spasticity and/or optic neuropathy. Brain 2012; 135: 2980-93.

23 Hewamadduma CA, Hoggard N, O'Malley R, et al. Novel genotype-phenotype and MRI correlations in a large cohort of patients with SPG7 mutations. Neurol Genet 2018; 4: e279.

24 Choquet K, Tetreault M, Yang S, et al. SPG7 mutations explain a significant proportion of French Canadian spastic ataxia cases. Eur J Hum Genet 2016; 24: 1016-21.

25 van Gassen KL, van der Heijden CD, de Bot ST, et al. Genotype-phenotype correlations in spastic paraplegia type 7: a study in a large Dutch cohort. Brain 2012; 135: 2994-3004.

26 Pfeffer G, Gorman GS, Griffin H, et al. Mutations in the SPG7 gene cause chronic progressive external ophthalmoplegia through disordered mitochondrial DNA maintenance. Brain 2014; 137: $1323-36$

27 McDermott CJ, Taylor RW, Hayes C, et al. Investigation of mitochondrial function in hereditary spastic paraparesis. Neuroreport 2003; 14: 485-88.

28 Wilkinson PA, Crosby AH, Turner C, et al. A clinical, genetic and biochemical study of SPG7 mutations in hereditary spastic paraplegia. Brain 2004; 127: 973-80.

29 Arnoldi A, Tonelli A, Crippa F, et al. A clinical, genetic, and biochemical characterization of SPG7 mutations in a large cohort of patients with hereditary spastic paraplegia. Hum Mutat 2008; 29: $522-31$

30 Sanchez-Ferrero E, Coto E, Beetz C, et al. SPG7 mutational screening in spastic paraplegia patients supports a dominant effect for some mutations and a pathogenic role for p.A510V. Clin Genet 2013; 83: 257-62.

31 Roxburgh RH, Marquis-Nicholson R, Ashton F, et al. The p.Ala510Val mutation in the SPG7 (paraplegin) gene is the most common mutation causing adult onset neurogenetic disease in patients of British ancestry. J Neurol 2013; 260: 1286-94.

32 McDermott CJ, Dayaratne RK, Tomkins J, et al. Paraplegin gene analysis in hereditary spastic paraparesis (HSP) pedigrees in northeast England. Neurology 2001; 56: 467-71.

33 Warnecke T, Duning T, Schirmacher A, et al. A novel splice site mutation in the SPG7 gene causing widespread fiber damage in homozygous and heterozygous subjects. Mov Disord 2010; 25: 413-20.

34 Dong EL, Wang C, Wu S, et al. Clinical spectrum and genetic landscape for hereditary spastic paraplegias in China. Mol Neurodegener 2018; 13: 36

35 Arnoldi A, Crimella C, Tenderini E, et al. Clinical phenotype variability in patients with hereditary spastic paraplegia type 5 associated with CYP7B1 mutations. Clin Genet 2012; 81: 150-57

36 Schols L, Rattay TW, Martus P, et al. Hereditary spastic paraplegia type 5: natural history, biomarkers and a randomized controlled trial. Brain 2017; 140: 3112-27.

37 Copp AJ, Stanier P, Greene NDE. Neural tube defects: recent advances, unsolved questions, and controversies. Lancet Neurol 2013; 12: $799-10$

38 van Es MA, Hardiman O, Chio A, et al. Amyotrophic lateral sclerosis. Lancet 2017; 390: 2084-98.

39 Psimaras D, Tafani C, Ducray F, et al. Bevacizumab in late-onset radiation-induced myelopathy. Neurology 2016; 86: 454-57.
40 Hedera P, Eldevik OP, Maly P, Rainier S, Fink JK. Spinal cord magnetic resonance imaging in autosomal dominant hereditary spastic paraplegia. Neuroradiology 2005; 47: 730-734.

41 Hobson GM, Garbern JY. Pelizaeus-Merzbacher disease, Pelizaeus-Merzbacher-like disease 1, and related hypomyelinating disorders. Semin Neurol 2012; 32: 62-67.

42 Marelli C, Salsano E, Politi LS, Labauge P. Spinal cord involvemen in adult-onset metabolic and genetic diseases. J Neurol Neurosurg Psychiatry 2018; 90: 211-18

43 Pascual B, de Bot ST, Daniels MR, et al. "Ears of the lynx" MRI sign is associated with SPG11 and SPG15 hereditary spastic paraplegia. Am J Neuroradiol 2019; 40: 199-203.

44 Martinuzzi A, Montanaro D, Vavla M, et al. Clinical and paraclinical indicators of motor system impairment in hereditary spastic paraplegia: a pilot study. PLoS One 2016; 11: e0153283

45 Toft A, Birk S, Ballegaard M, et al. Peripheral neuropathy in hereditary spastic paraplegia caused by REEP1 variants. J Neurol 2019; 266: 735-44.

46 Burguez D, Polese-Bonatto M, Scudeiro LAJ, et al. Clinical and molecular characterization of hereditary spastic paraplegias: a next-generation sequencing panel approach. J Neurol Sci 2017; 383: $18-25$

47 Hensiek A, Kirker S, Reid E. Diagnosis, investigation and management of hereditary spastic paraplegias in the era of next-generation sequencing. J Neurol 2015; 262: 1601-12.

48 Boone PM, Liu P, Zhang F, et al. Alu-specific microhomology-mediated deletion of the final exon of SPAST in three unrelated subjects with hereditary spastic paraplegia. Genet Med 2011; 13: 582-92.

49 Soong BW, Morrison PJ. Spinocerebellar ataxias. Handb Clin Neurol 2018; 155: 143-74.

50 Synofzik M, Nemeth AH. Recessive ataxias. Handb Clin Neurol 2018; 155: 73-89.

51 Bereznyakova O, Dupre N. Spastic ataxias. Handb Clin Neurol 2018; 155: 191-203.

52 Lynch DS, Wade C, Paiva ARB, et al. Practical approach to the diagnosis of adult-onset leukodystrophies: an updated guide in the genomic era. J Neurol Neurosurg Psychiatry 2019; 90: 543-54.

53 Travaglini L, Aiello C, Stregapede F, et al. The impact of next-generation sequencing on the diagnosis of pediatric-onse hereditary spastic paraplegias: new genotype-phenotype correlations for rare HSP-related genes. Neurogenetics 2018; 19: $111-21$

54 Karlstrom H, Brooks WS, Kwok JB, et al. Variable phenotype of Alzheimer's disease with spastic paraparesis. J Neurochem 2008; 104: 573-83.

55 Faber I, Prota JR, Martinez AR, Lopes-Cendes I, Franca MCJ. A new phenotype associated with homozygous GRN mutations: complicated spastic paraplegia. Eur J Neurol 2017; 24: e3-4.

56 Khalifa M, Naffaa L. Exome sequencing reveals a novel WDR45 frameshift mutation and inherited POLR3A heterozygous variants in a female with a complex phenotype and mixed brain MRI findings. Eur J Med Genet 2015; 58: 381-86.

57 Song Y, Liu Y, Zhang N, Long L. Spinocerebellar ataxia type 3/Machado-Joseph disease manifested as spastic paraplegia: A clinical and genetic study. Exp Ther Med 2015; 9: 417-20.

58 Pedroso JL, de Souza PV, Pinto WB, et al. SCA1 patients may present as hereditary spastic paraplegia and must be included in spastic-ataxias group. Parkinsonism Relat Disord 2015; 21: 1243-46.

59 Wilkinson PA, Bradley JL, Warner TT. Friedreich's ataxia presenting as an isolated spastic paraparesis. J Neurol Neurosurg Psychiatry 2001; 71: 709 .

60 Maris T, Androulidakis EJ, Tzagournissakis M, Papavassiliou S, Moser H, Plaitakis A. X-linked adrenoleukodystrophy presenting as neurologically pure familial spastic paraparesis. Neurology 1995 ; 45: 1101-04.

61 Tsang JP, Poon WL, Luk HM, et al. Arginase deficiency with new phenotype and a novel mutation: contemporary summary. Pediatr Neurol 2012; 47: 263-69.

62 Saute JA, Giugliani R, Merkens LS, Chiang JP, DeBarber AE, de Souza CF. Look carefully to the heels! A potentially treatable cause of spastic paraplegia. J Inherit Metab Dis 2015; 38: 363-64. 
63 Furukawa Y, Graf WD, Wong H, Shimadzu M, Kish SJ. Dopa-responsive dystonia simulating spastic paraplegia due to tyrosine hydroxylase (TH) gene mutations. Neurology 2001; 56: 260-63.

64 Kasim S, Moo LR, Zschocke J, Jinnah HA. Phenylketonuria presenting in adulthood as progressive spastic paraparesis with dementia. J Neurol Neurosurg Psychiatry 2001; 71: 795-97.

65 Wolf B. Biotinidase deficiency should be considered in individuals exhibiting myelopathy with or without and vision loss. Mol Genet Metab 2015; 116: 113-18.

66 Huemer M, Diodato D, Schwahn B, et al. Guidelines for diagnosis and management of the cobalamin-related remethylation disorders cblC, cblD, cblE, cblF, cblG, cblJ and MTHFR deficiency. $J$ Inherit Metab Dis 2017; 40: 21-48.

67 Lossos A, Teltsh O, Milman T, et al. Severe methylenetetrahydrofolate reductase deficiency: clinical clues to a potentially treatable cause of adult-onset hereditary spastic paraplegia. JAMA Neurol 2014; 71: 901-04.

68 Wang Y, Smith C, Parboosingh JS, Khan A, Innes M, Hekimi S. Pathogenicity of two COQ7 mutations and responses to 2,4-dihydroxybenzoate bypass treatment. J Cell Mol Med 2017; 21: 2329-43.

69 Scheuer KH, Svenstrup K, Jennum P, et al. Double-blind crossover trial of gabapentin in SPG4-linked hereditary spastic paraplegia. Eur J Neurol 2007; 14: 663-66.

70 Bereau M, Anheim M, Chanson JB, et al. Dalfampridine in hereditary spastic paraplegia: a prospective, open study. J Neurol 2015; 262: 1285-88.

71 Margetis K, Korfias S, Boutos N, et al. Intrathecal baclofen therapy for the symptomatic treatment of hereditary spastic paraplegia. Clin Neurol Neurosurg 2014; 123: 142-45.

72 de Niet M, de Bot ST, van de Warrenburg BP, Weerdesteyn V, Geurts AC. Functional effects of botulinum toxin type-A treatment and subsequent stretching of spastic calf muscles: a study in patients with hereditary spastic paraplegia. J Rehabil Med 2015; 47: 147-53.

73 Marsden J, Stevenson V, McFadden C, Swain I, Taylor P. The effects of functional electrical stimulation on walking in hereditary and spontaneous spastic paraparesis. Neuromodulation 2013; 16: 256-60.

74 Ardolino G, Bocci T, Nigro M, et al. Spinal direct current stimulation (tsDCS) in hereditary spastic paraplegias (HSP): a sham-controlled crossover study. J Spinal Cord Med 2018; published online Dec 3. DOI:10.1080/10790268.2018.1543926.

75 Nonnekes J, van Lith B, van de Warrenburg BP, Weerdesteyn V, Geurts ACH. Pathophysiology, diagnostic work-up and management of balance impairments and falls in patients with hereditary spastic paraplegia. J Rehabil Med 2017; 49: 369-77.

76 Martino G, Ivanenko Y, Serrao M, et al. Differential changes in the spinal segmental locomotor output in hereditary spastic paraplegia. Clin Neurophysiol 2018; 129: 516-25.

77 Nonnekes J, Oude Nijhuis LB, de Niet M, et al. StartReact restores reaction time in HSP: evidence for subcortical release of a motor program. J Neurosci 2014; 34: 275-81.

78 Bertolucci F, Di Martino S, Orsucci D, et al. Robotic gait training improves motor skills and quality of life in hereditary spastic paraplegia. NeuroRehabilitation 2015; 36: 93-99.

79 Schneider SA, Beckinger VE, Moller B, Knupfer S, Hamann M, Deuschl G. Urinary symptoms, quality of life, and patient satisfaction in genetic and sporadic hereditary spastic paraplegia. J Neurol 2018; 266: 207-11.

80 Roll-Mecak A, Vale RD. Structural basis of microtubule severing by the hereditary spastic paraplegia protein spastin. Nature 2008; 451: 363-67.

81 Denton KR, Lei L, Grenier J, Rodionov V, Blackstone C, Li XJ. Loss of spastin function results in disease-specific axonal defects in human pluripotent stem cell-based models of hereditary spastic paraplegia. Stem Cells 2014; 32: 414-23.

82 Ciccarelli FD, Proukakis C, Patel H, et al. The identification of a conserved domain in both spartin and spastin, mutated in hereditary spastic paraplegia. Genomics 2003; 81: 437-41.

83 Sherwood NT, Sun Q, Xue M, Zhang B, Zinn K. Drosophila spastin regulates synaptic microtubule networks and is required for normal motor function. PLoS Biol 2004; 2: e429.
84 Brill MS, Kleele T, Ruschkies L, et al. Branch-specific microtubule destabilization mediates axon branch loss during neuromuscular synapse elimination. Neuron 2016; 92: 845-56.

85 Jardin N, Giudicelli F, Ten Martin D, et al. BMP- and neuropilin 1-mediated motor axon navigation relies on spastin alternative translation. Development 2018; 145: dev162701.

86 Tarrade A, Fassier C, Courageot S, et al. A mutation of spastin is responsible for swellings and impairment of transport in a region of axon characterized by changes in microtubule composition. Hum Mol Genet 2006; 15: 3544-58.

87 Julien C, Lissouba A, Madabattula S, et al. Conserved pharmacological rescue of hereditary spastic paraplegia-related phenotypes across model organisms. Hum Mol Genet 2016; 25: 1088-99.

88 Wali G, Sue CM, Mackay-Sim A. Patient-derived stem cell models in SPAST HSP: disease modelling and drug discovery. Brain Sci 2018; 8: 142

89 Orso G, Martinuzzi A, Rossetto MG, Sartori E, Feany M, Daga A. Disease-related phenotypes in a Drosophila model of hereditary spastic paraplegia are ameliorated by treatment with vinblastine. J Clin Invest 2005; 115: 3026-34.

90 Fassier C, Tarrade A, Peris L, et al. Microtubule-targeting drugs rescue axonal swellings in cortical neurons from spastin knockout mice. Dis Model Mech 2013; 6: 72-83.

91 Havlicek S, Kohl Z, Mishra HK, et al. Gene dosage-dependent rescue of HSP neurite defects in SPG4 patients' neurons. Hum Mol Genet 2014; 23: 2527-41.

92 Mignarri A, Malandrini A, Del Puppo M, et al. Treatment of SPG5 with cholesterol-lowering drugs. J Neurol 2015; 262: 2783-85.

93 Marelli C, Lamari F, Rainteau D, et al. Plasma oxysterols: biomarkers for diagnosis and treatment in spastic paraplegia type 5 . Brain 2018; 141: 72--84.

94 Rismanchi N, Soderblom C, Stadler J, Zhu PP, Blackstone C. Atlastin GTPases are required for Golgi apparatus and ER morphogenesis. Hum Mol Genet 2008; 17: 1591-604.

95 Ulengin I, Park JJ, Lee TH. ER network formation and membrane fusion by atlastin1/SPG3A disease variants. Mol Biol Cell 2015; 26: 1616-28.

96 Beetz C, Koch N, Khundadze M, et al. A spastic paraplegia mouse model reveals REEP1-dependent ER shaping. J Clin Invest 2013; 123: 4273-82.

97 Chang J, Lee S, Blackstone C. Protrudin binds atlastins and endoplasmic reticulum-shaping proteins and regulates network formation. Proc Natl Acad Sci USA 2013; 110: 14954-59.

98 Renvoise B, Malone B, Falgairolle M, et al. Reep1 null mice reveal a converging role for hereditary spastic paraplegia proteins in lipid droplet regulation. Hum Mol Genet 2016; 25: 5111-25.

99 Klemm RW, Norton JP, Cole RA, et al. A conserved role for atlastin GTPases in regulating lipid droplet size. Cell Rep 2013; 3: 1465-75.

100 Zhu PP, Denton KR, Pierson TM, Li XJ, Blackstone C. Pharmacologic rescue of axon growth defects in a human iPSC model of hereditary spastic paraplegia SPG3A. Hum Mol Genet 2014; 23: 5638-48.

101 Ferreirinha F, Quattrini A, Pirozzi M, et al. Axonal degeneration in paraplegin-deficient mice is associated with abnormal mitochondria and impairment of axonal transport. J Clin Invest 2004; 113: 231-42.

102 Shanmughapriya S, Rajan S, Hoffman NE, et al. SPG7 is an essential and conserved component of the mitochondrial permeability transition pore. Mol Cell 2015; 60: 47-62.

103 Almontashiri NA, Chen HH, Mailloux RJ, et al. SPG7 variant escapes phosphorylation-regulated processing by AFG3L2, elevates mitochondrial ROS, and is associated with multiple clinical phenotypes. Cell Rep 2014; 7: 834-47.

104 Pirozzi M, Quattrini A, Andolfi G, et al. Intramuscular viral delivery of paraplegin rescues peripheral axonopathy in a model of hereditary spastic paraplegia. J Clin Invest 2006; 116: 202-08.

105 Hirst J, Borner GH, Edgar J, et al. Interaction between AP-5 and the hereditary spastic paraplegia proteins SPG11 and SPG15. Mol Biol Cell 2013; 24: 2558-69.

106 Varga RE, Khundadze M, Damme M, et al. In vivo evidence for lysosome depletion and impaired autophagic clearance in hereditary spastic paraplegia type SPG11. PLoS Genet 2015; 11: e1005454. 
107 Branchu J, Boutry M, Sourd L, et al. Loss of spatacsin function alters lysosomal lipid clearance leading to upper and lower motor neuron degeneration. Neurobiol Dis 2017; 102`21-37.

108 Chang J, Lee S, Blackstone C. Spastic paraplegia proteins spastizin and spatacsin mediate autophagic lysosome reformation. J Clin Invest 2014; 124: 5249-62.

109 Vantaggiato C, Panzeri E, Castelli M, et al. ZFYVE26/SPASTIZIN and SPG11/SPATACSIN mutations in hereditary spastic paraplegia types AR-SPG15 and AR-SPG11 have different effects on autophagy and endocytosis. Autophagy 2019; 15: 34-57.
110 Mishra HK, Prots I, Havlicek S, et al. GSK3ss-dependent dysregulation of neurodevelopment in SPG11-patient induced pluripotent stem cell model. Ann Neurol 2016; 79: 826-40.

111 Pozner T, Schray A, Regensburger M, et al. Tideglusib rescues neurite pathology of SPG11 iPSC derived cortical neurons. Front Neurosci 2018; 12: 914.

(C) 2019 Elsevier Ltd. All rights reserved. 